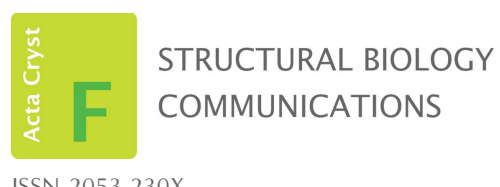

ISSN 2053-230X

\section{The use of noncrystallographic symmetry averaging to solve structures from data affected by perfect hemihedral twinning}

\author{
Charles Sabin and Pavel Plevka*
}

Received 9 November 2015

Accepted 15 January 2016

Edited by W. N. Hunter, University of Dundee, Scotland

Keywords: hemihedral perfect twinning; noncrystallographic symmetry averaging; molecular replacement; virus structure; symmetry; detwinning; twin domain; merohedral twinning; mask envelope.

PDB reference: Aichi virus 1, 5aoo

Supporting information: this article has supporting information at journals.iucr.org/f
Central European Institute of Technology - Masaryk University, Kamenice 653/25, 62500 Brno, Czech Republic. *Correspondence e-mail: pavel.plevka@ceitec.muni.cz

Hemihedral twinning is a crystal-growth anomaly in which a specimen is composed of two crystal domains that coincide with each other in three dimensions. However, the orientations of the crystal lattices in the two domains differ in a specific way. In diffraction data collected from hemihedrally twinned crystals, each observed intensity contains contributions from both of the domains. With perfect hemihedral twinning, the two domains have the same volumes and the observed intensities do not contain sufficient information to detwin the data. Here, the use of molecular replacement and of noncrystallographic symmetry (NCS) averaging to detwin a $2.1 \AA$ resolution data set for Aichi virus 1 affected by perfect hemihedral twinning is described. The NCS averaging enabled the correction of errors in the detwinning introduced by the differences between the molecular-replacement model and the crystallized structure. The procedure permitted the structure to be determined from a molecular-replacement model that had $16 \%$ sequence identity and a $1.6 \AA$ r.m.s.d. for $\mathrm{C}^{\alpha}$ atoms in comparison to the crystallized structure. The same approach could be used to solve other data sets affected by perfect hemihedral twinning from crystals with NCS.

\section{Introduction}

Aichi virus 1 (AiV1) is a member of the Kobuvirus genus belonging to the Picornaviridae family of small non-enveloped viruses (Yamashita et al., 1991). The outer diameter of the virion is about $300 \AA$. The capsid of AiV1 is composed of 60 copies of each of the three capsid proteins VP0, VP1 and VP3 organized with icosahedral symmetry. The AiV1 genome is a single-stranded positive-sense RNA 8251 nucleotides in length (Yamashita et al., 1998). Human infection by AiV1 can result in gastroenteritis (Yamashita et al., 1998).

Twinning is a crystal-growth anomaly in which a crystal specimen is composed of domains whose orientations give rise to overlapping diffraction patterns (Redinbo \& Yeates, 1993; Yeates \& Fam, 1999; Chandra et al., 1999; Helliwell, 2008; Grainger, 1969). In hemihedral twinning, the specimen is composed of two domains whose crystal lattices coincide with each other in three dimensions. Since the real-space lattices of the two domains coincide, the reciprocal lattices of the domains lie on top of each other (Yeates, 1997; Parsons, 2003). The domain sizes in the twinned crystals are presumed to be large compared with the coherence length of the X-ray beam, so the waves scattered from the separate domains do not interfere. Thus, in hemihedral twinning, each observed intensity $I_{\mathrm{obs}}(\mathbf{h})$ is a weighted sum of the intensities of the reflections from the two domains $I\left(\mathbf{h}_{1}\right)$ and $I\left(\mathbf{h}_{2}\right)$, 


$$
\begin{aligned}
& I_{\text {obs }}\left(\mathbf{h}_{1}\right)=(1-\alpha) I\left(\mathbf{h}_{1}\right)+\alpha I\left(\mathbf{h}_{2}\right), \\
& I_{\text {obs }}\left(\mathbf{h}_{2}\right)=\alpha I\left(\mathbf{h}_{1}\right)+(1-\alpha) I\left(\mathbf{h}_{2}\right) .
\end{aligned}
$$

The twinning fraction $(\alpha)$ represents the part of the volume of the specimen occupied by the domain in the arbitrarily selected 'first' orientation. The domain in the 'second' orien- tation occupies the remaining $(1-\alpha)$ part of the specimen volume. The case of $\alpha=0$ corresponds to an untwinned specimen. Cases where $0<\alpha<<0.5$ are referred to as 'partial twinning' and cases where $\alpha$ approaches 0.5 as 'perfect twinning' (Parsons, 2003; Yeates, 1997). The two domains are related by the twinning operator, but not by their crystallographic symmetry (Yeates, 1997; Parsons, 2003).
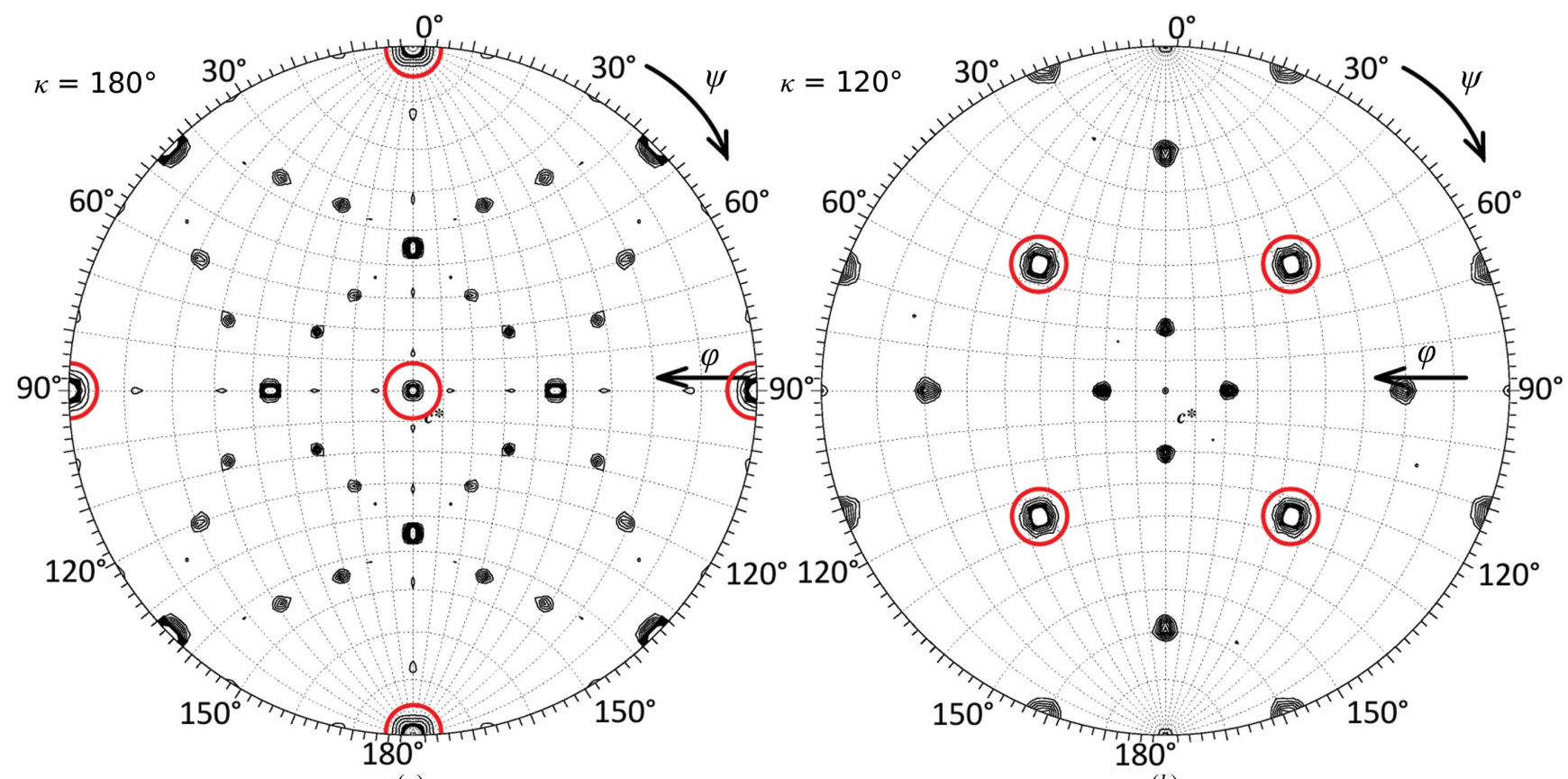

(a)

(b)

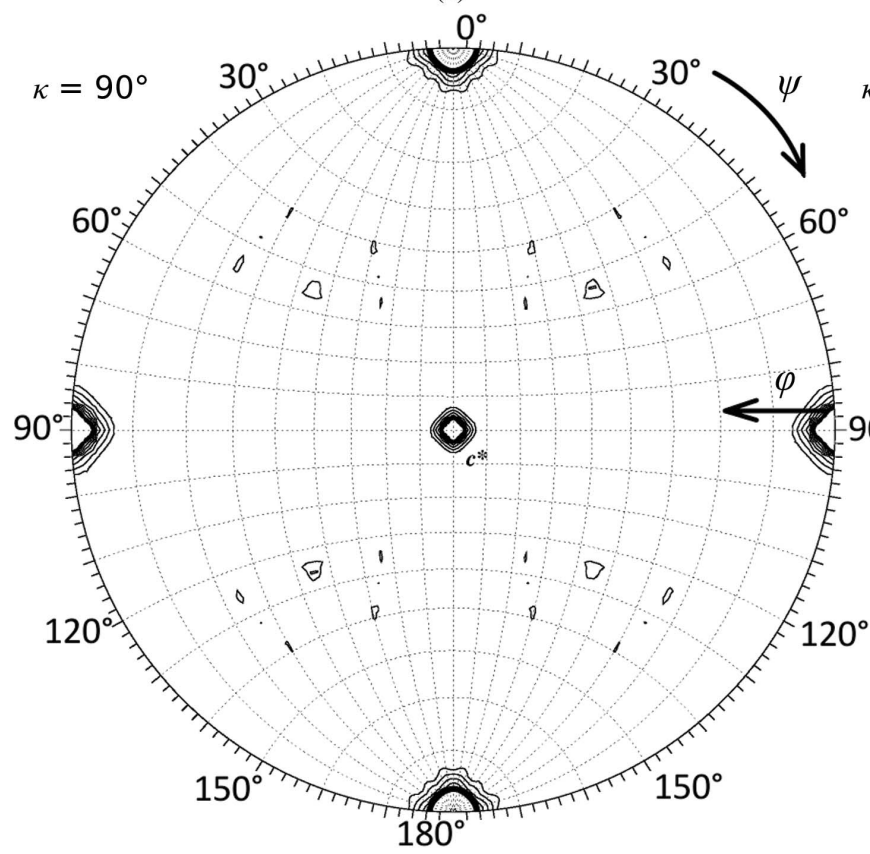

(c)

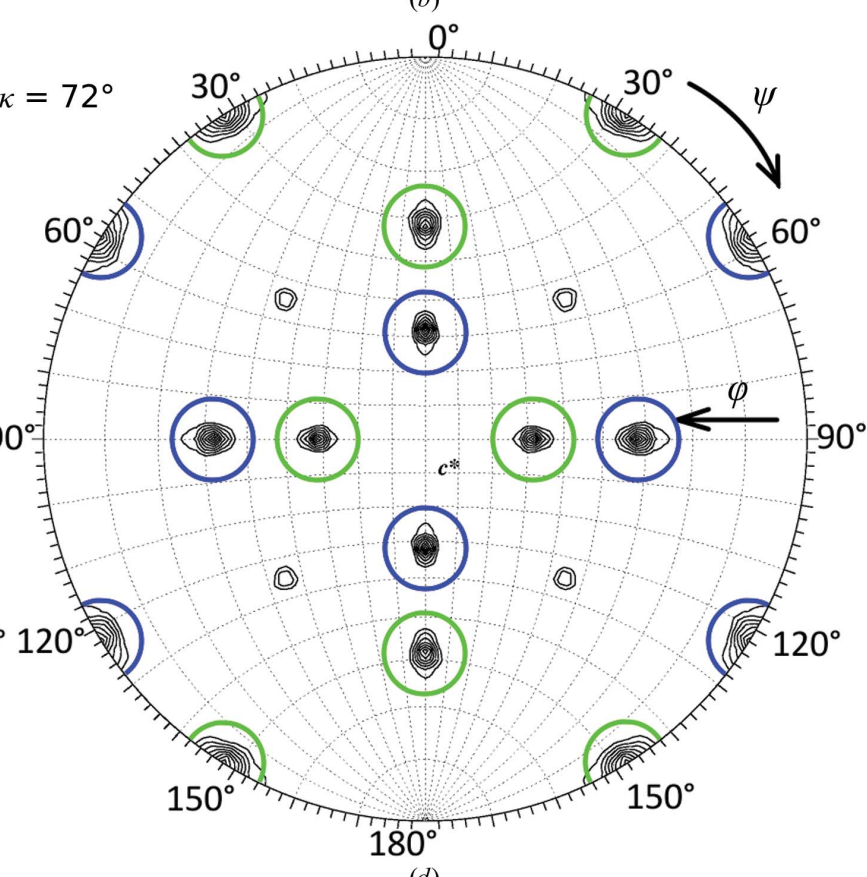

(d)

Figure 1

Sections of the rotation function calculated from AiV1 diffraction data processed in space group I23. Stereographic plots of $(a) \kappa=180^{\circ},(b) \kappa=120^{\circ}$, (c) $\kappa=90^{\circ}$ and $(d) \kappa=72^{\circ}$ rotation-function sections were calculated using $12-7 \AA$ resolution AiV1 diffraction data and a $150 \AA$ radius of integration. The plots were contoured in $0.5 \sigma$ increments of the rotation-function values starting from $1.0 \sigma$. Peaks of shared crystallographic and icosahedral symmetry are highlighted with red circles in $(a)$ and $(b)$. All of the remaining non-noise peaks belong to NCS symmetry. Fivefold symmetry peaks corresponding to the twin domains are differentiated by blue and green circles in $(d)$. 
Table 1

Analysis of rotational symmetry in AiV1 I23 diffraction data.

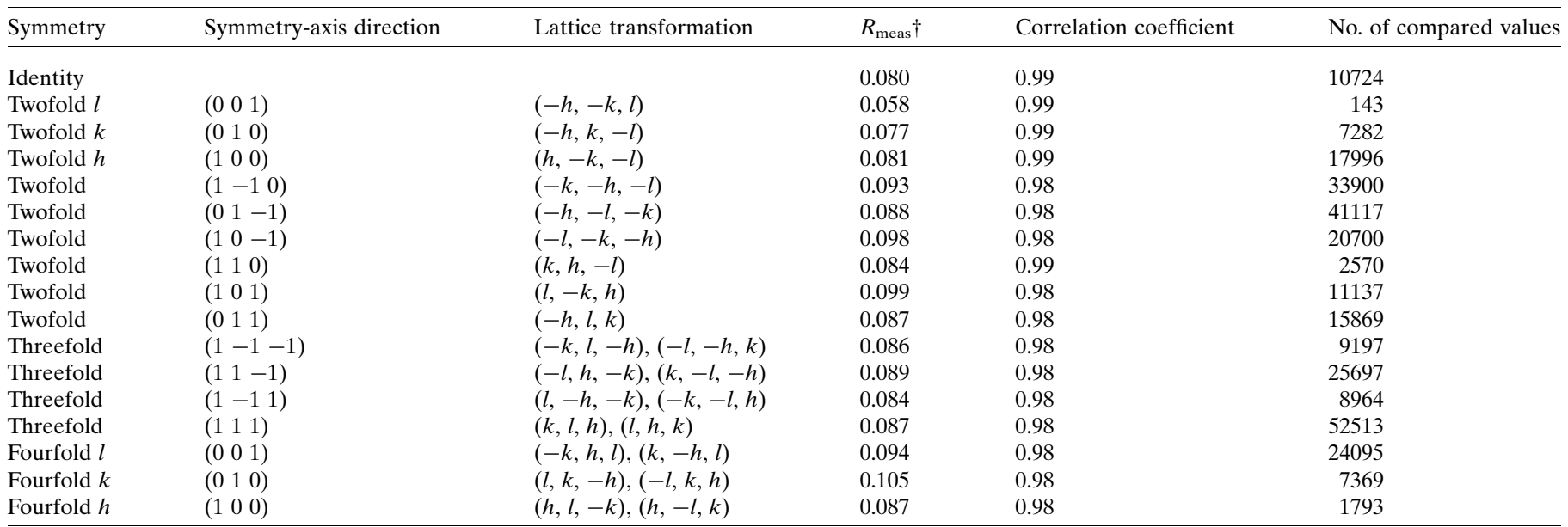

$\dagger R_{\text {meas }}=\sum_{h k l}\{N(h k l) /[N(h k l)-1]\}^{1 / 2} \sum_{i}\left|I_{i}(h k l)-\langle I(h k l)\rangle\right| / \sum_{h k l} \sum_{i} I_{i}(h k l)$.

Partial hemihedral twinning does not obscure the true crystallographic symmetry because the pairs of reflections related by the twinning operator have different intensities (Parsons, 2003). A statistical analysis of the observed intensities can be used to estimate the twinning fraction $\alpha$. The true crystallographic intensities can be calculated, using the $\alpha$ value, based on (3) and (4) (Grainger, 1969; Yeates, 1997):

$$
\begin{gathered}
I\left(\mathbf{h}_{1}\right)=\left[(1-\alpha) I_{\mathrm{obs}}\left(\mathbf{h}_{1}\right)-\alpha I_{\mathrm{obs}}\left(\mathbf{h}_{2}\right)\right] /(1-2 \alpha), \\
I\left(\mathbf{h}_{2}\right)=\left[-\alpha I_{\mathrm{obs}}\left(\mathbf{h}_{1}\right)+(1-\alpha) I_{\mathrm{obs}}\left(\mathbf{h}_{2}\right)\right] /(1-2 \alpha) .
\end{gathered}
$$

As the twinning fraction $\alpha$ approaches 0.5 the term $(1-2 \alpha)$ approaches zero, and the true crystallographic intensities cannot be accurately calculated based on (3) and (4). With a perfect twin (twin fraction $\alpha=0.5$ ) the two reflections related by the twin law contribute equally to both of the observed intensities related by the twinning operator: $I_{\text {obs }}\left(\mathbf{h}_{1}\right)=0.5 I\left(\mathbf{h}_{1}\right)$ $+0.5 I\left(\mathbf{h}_{2}\right)$ and $I_{\text {obs }}\left(\mathbf{h}_{2}\right)=0.5 I\left(\mathbf{h}_{1}\right)+0.5 I\left(\mathbf{h}_{2}\right)$ (Yeates, 1997). Therefore, the symmetry of the twinning operation is superimposed on top of the actual Laue symmetry and the apparent Laue group is of a higher order than the actual Laue group (Supplementary Fig. S1).

Here, we report the structure determination of an AiV1 virion based on diffraction data affected by perfect hemihedral twinning. The structure of AiV1 and its implications for the infection process and the design of antiviral compounds will be described elsewhere. This paper focuses on the utilization of noncrystallographic symmetry (NCS) averaging to solve a structure from a data set affected by perfect hemihedral twinning.

\section{Materials and methods}

\subsection{Virus growth and purification}

Green monkey kidney (GMK) cells were grown on $150 \mathrm{~mm}$ diameter plates to $70 \%$ confluency in Dulbecco's Modified Eagle's Medium (DMEM) supplemented with 10\% foetal bovine serum (FBS; Sigma-Aldrich). 60 plates of green monkey kidney cells were infected with AiV1 with a multiplicity of infection (MOI) of 0.1 (GenBank AB040749.1; obtained from Dr A. Michael Lindberg, Linnaeus University, Sweden) and incubated at $37^{\circ} \mathrm{C}$ and $5 \% \mathrm{CO}_{2}$ until the cytopathic effect was observed. Both cells and virus-containing supernatant were harvested. The cells were pelleted by centrifugation at $4500 \mathrm{rev} \mathrm{min}{ }^{-1}$ at $4^{\circ} \mathrm{C}$ for $15 \mathrm{~min}$ in a Beckman JA-10 rotor and lysed by freezing and thawing three times followed by Dounce homogenization. The cell debris was removed by centrifugation at $10000 \mathrm{rev} \mathrm{min}^{-1}$ for $15 \mathrm{~min}$ in a Beckman Coulter JA-10 rotor. The virus-containing supernatant was pooled with the previously harvested viruscontaining tissue-culture medium. Polyethylene glycol 8000 and $\mathrm{NaCl}$ were added to final concentrations of $5 \%$ and $0.5 \mathrm{M}$, respectively. The virus was precipitated by overnight incubation at $4^{\circ} \mathrm{C}$ with gentle shaking. The precipitate was pelleted by $15 \mathrm{~min}$ centrifugation at $9500 \mathrm{rev} \min ^{-1}$ and $4^{\circ} \mathrm{C}$ using a Beckman Coulter JA-10 rotor. The pellet was resuspended in buffer $A$ [0.25 $M$ 4-(2-hydroxyethyl)-1-piperazineethanesulfonic acid (HEPES) pH 7.5, $0.25 \mathrm{M} \mathrm{NaCl}$ ] and treated with $\mathrm{MgCl}_{2}$ (final concentration $0.005 \mathrm{M}$ ), DNase (final concentration $10 \mu \mathrm{g} \mathrm{ml}^{-1}$ ) and RNase (final concentration $10 \mu \mathrm{g} \mathrm{ml}^{-1}$ ) for $30 \mathrm{~min}$ at room temperature followed by trypsin (final concentration $80 \mu \mathrm{g} \mathrm{ml}^{-1}$ ) digestion for $10 \mathrm{~min}$ at $37^{\circ} \mathrm{C}$. Subsequently, ethylendiaminetetraacetic acid and Nonidet P-40 were added to final concentrations of $0.015 M$ and $1 \%$, respectively. The solution was centrifuged at $4500 \mathrm{rev} \mathrm{min}{ }^{-1}$ for $10 \mathrm{~min}$ in a Beckman Coulter JA-10 rotor and the resulting pellet was discarded. The clarified supernatant was layered over a $30 \%(w / v)$ sucrose cushion in buffer $A$ and centrifuged in a Beckman Coulter 50.2 Ti rotor at $48000 \mathrm{rev} \mathrm{min}^{-1}$ for $2 \mathrm{~h}$ at $10^{\circ} \mathrm{C}$. After centrifugation, the supernatant was discarded and the pellet was resuspended in $1 \mathrm{ml}$ buffer $A$. The virus suspension was layered onto a $10-40 \%$ tartrate gradient and centrifuged in a Beckman Coulter SW 41 Ti rotor for 90 min at $36000 \mathrm{rev} \min ^{-1}$ and $4^{\circ} \mathrm{C}$. The virus band was collected using a syringe with a needle. The virus was transferred to buffer $A$ using repeated concentration and dilution steps of the virus 
Twinning statistics for the AiV1 diffraction data.

\begin{tabular}{|c|c|c|c|c|c|c|c|}
\hline & \multicolumn{7}{|c|}{ Twinning test } \\
\hline & \multirow[b]{2}{*}{$L$ test } & \multicolumn{3}{|c|}{ Mean acentric moments of $I$ (moment No.) } & \multicolumn{3}{|c|}{ Mean centric moments of $I$ (moment No.) } \\
\hline & & 2 & 3 & 4 & 2 & 3 & 4 \\
\hline Untwinned expected value & 0.50 & 2.0 & 6.0 & 24.0 & 3.0 & 15.0 & 105.0 \\
\hline Twinned expected value & 0.38 & 1.5 & 3.0 & 7.5 & 2.0 & 6.0 & 24.0 \\
\hline$I 23$ data $(72.0-2.3 \AA)$ & 0.46 & 2.1 & 6.2 & 25.4 & 2.8 & 11.8 & 71.3 \\
\hline$P 4_{2} 32$ data $(68.0-3.5 \AA)$ & 0.23 & 1.2 & 1.7 & 2.7 & 1.4 & 1.9 & 4.4 \\
\hline
\end{tabular}

solution in centrifugal concentrators with a $100 \mathrm{kDa}$ cutoff membrane. The concentration of the purified virus was measured in a spectrophotometer using an absorption coefficient of $7.25 \mathrm{mg} \mathrm{ml}^{-1} \mathrm{~cm}^{-1}$ at $260 \mathrm{~nm}$.

\subsection{Crystallization and data collection}

The AiV1 crystals were grown using the hanging-drop vapour-diffusion method at $20^{\circ} \mathrm{C}$ with a well solution consisting of $0.05 M$ cadmium sulfate, $0.1 M$ HEPES $\mathrm{pH}$ 7.5, 1.0 $M$ sodium acetate. Crystallization drops were prepared by mixing $1 \mu \mathrm{l}$ well solution with an equal volume of the virus at a concentration of $3 \mathrm{mg} \mathrm{ml}^{-1}$ in buffer $A$. The crystals formed within one month. For diffraction experiments, the crystals were directly vitrified in liquid nitrogen without presoaking in any cryoprotectant. A single-crystal diffraction data set was collected using a Pilatus $36 \mathrm{M} 100 \mathrm{~Hz}$ detector on beamline I03 at Diamond Light Source, UK. An oscillation range of $0.1^{\circ}$ and a wavelength of $0.976 \AA$ were used for data collection. The diffraction pattern extended to a resolution of $2.1 \AA$. The diffraction images were processed and scaled using $X D S$ and AIMLESS from the CCP4 software package (Kabsch, 2010; Winn et al., 2011).

\subsection{Data deposition}

The model of AiV1 together with the observed structurefactor amplitudes and intensities was deposited in the Protein Data Bank as entry 5aoo. Detwinned structure-factor amplitudes and phases calculated from the refined model and refined by 30 cycles of NCS averaging were also deposited.

\section{Results and discussion}

\subsection{Detection of twinning in the AiV 1 diffraction data}

The AiV1 crystal diffracted to a resolution of $2.1 \AA$. The diffraction pattern was compatible with a body-centred lattice and had 432 point symmetry (Table 1; Evans, 2006). Since there was no indication of systematic absences along the fourfold axis (reflections $h 00: h=4 n$ ) the space group was identified as I432. The presence of the crystallographic and NCS axes belonging to the symmetry of the crystallized AiV1 virions is shown in the rotation-function sections for twofold, threefold, fourfold and fivefold symmetry (Fig. 1; Tong \& Rossmann, 1990). Statistical analysis did not indicate twinning (Table 2; Evans, 2006; Padilla \& Yeates, 2003). However, no molecular-replacement solution with good packing was obtainable in space group I432 (Supplementary Fig. 2a). The unit-cell volume and the rotation-function plots indicated that a crystal with the same unit-cell parameters but with $I 23$ symmetry (a subset of $I 432$ symmetry) would allow the packing of AiV1 virions (Supplementary Fig. S2b). This provided evidence that the crystal may be hemihedrally twinned, with the two domains composed of $I 23$ unit cells rotated $90^{\circ}$ relative to each other. Since the fourfold symmetry axes owing to the twinning had a similar $R_{\text {meas }}$ as the twofold and threefold axes belonging to the $I 23$ crystallographic symmetry (Table 1) the data were perfectly hemihedrally twinned.

\subsection{Data processing}

For the twinning analyses and the calculation of rotation functions, the AiV1 diffraction images were processed in space group $I 23$ to a resolution of $2.3 \AA$ using XDS (Kabsch, 2010). However, after determining that the diffraction data were affected by perfect hemihedral twinning, the diffraction images were reprocessed in space group I432. Taking advantage of the higher symmetry enabled us to obtain a data set that was more than $90 \%$ complete from the first 160 diffraction images (total rotation range of $16^{\circ}$ ). The data could be processed to a resolution of $2.1 \AA$ (Table 3). Subsequently, the diffraction data were expanded from I432 to $I 23$ using SFTOOLS from CCP4 (Winn et al., 2011).

\subsection{Molecular-replacement solution}

The twofold and threefold rotation-function plots indicated that a subset of the icosahedral twofold and threefold symmetry axes were aligned with the crystallographic cubic symmetry axes (Figs. $1 a$ and $1 b$ and Supplementary Fig. S2b). Icosahedral 532 symmetry elements can be aligned with those of 23 symmetry in two equivalent choices that are rotated $90^{\circ}$ relative to one another. However, both of the icosahedral orientations were present because the two crystal twin domains are related by a $90^{\circ}$ rotation (Figs. $1 a, 1 b$ and $1 d$ and Supplementary Fig. S2b). To verify the consistency of the particle in the standard orientation with the experimental data, values of the icosahedral locked rotation function with the twofold icosahedral axes aligned with the coordinate axes were calculated for symmetry rotated 0 and $90^{\circ}$ about the $z$ axis using the data processed in space group I23. Similar values of the locked rotation function at rotations of 0 and $90^{\circ}$ (4.7 and $4.3 \sigma$, respectively) verified that the data were affected by perfect hemihedral twinning. Superimposition of the 
Table 3

Diffraction data and structure-quality indicators.

Values in parentheses are for the highest resolution shell. Because of the perfect hemihedral twinning, the data were integrated and scaled in space group I432. A greater than $90 \%$ complete data set with a resolution of $2.1 \AA$ was obtained from the first 160 images $\left(0.1^{\circ}\right.$ oscillation per frame $)$ that were least affected by radiation damage. For refinement, the data were expanded to space group $I 23$.

\begin{tabular}{|c|c|c|c|c|}
\hline & \multicolumn{3}{|c|}{ Twinned crystal processed in space group } & \multirow{2}{*}{$\frac{\text { Primitive cell }}{\mathrm{P}_{2} 32}$} \\
\hline & $I 23$ & $I 432$ & $I 23$ expanded from $I 432$ & \\
\hline Unit-cell parameter $a(\AA)$ & 350.8 & 350.8 & & 351.1 \\
\hline No. of unique observations & 282853 (14471) & $186646(8521)$ & 367359 (16771) & $86648(4550)$ \\
\hline Observation multiplicity & $3.2(3.0)$ & $3.9(2.6)$ & & $2.9(2.9)$ \\
\hline Completeness $(\%)$ & $91.0(94.0)$ & $90.0(83.6)$ & & $93.6(94.1)$ \\
\hline$R$ factor & & & 0.33 & \\
\hline No. of protein atoms§ & & & 5791 & \\
\hline No. of water atoms§ & & & 147 & \\
\hline Average $B$ factor $\left(\AA^{2}\right)$ & & & 22.9 & \\
\hline \multicolumn{5}{|l|}{ Ramachandran statistics } \\
\hline Preferred regions (\%) & & & 95.7 & \\
\hline Allowed regions (\%) & & & 4.0 & \\
\hline
\end{tabular}

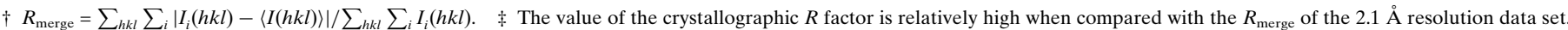

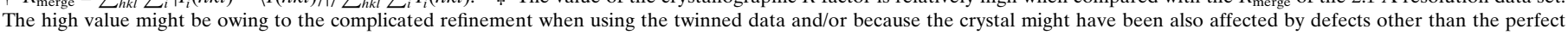
hemihedral twinning. $§$ Data are given for one icosahedral asymmetric unit. \ According to MolProbity (Chen et al., 2010).

Table 4

Comparison of molecular-replacement models.

The icosahedral asymmetric units were used as rigid bodies in all cases. $O$ (Jones et al., 1990) was used for superposition of the molecules. The cutoff for the inclusion of residues in the r.m.s.d calculation was $3.8 \AA$

\begin{tabular}{|c|c|c|c|c|c|c|c|c|c|c|}
\hline MR model (PDB code) & 1bev & $1 \mathrm{cov}$ & 1ev1 & $1 \mathrm{hxs}$ & $1 \mathrm{tmf}$ & $2 \mathrm{mev}$ & 2wff & $2 \times 5 i$ & $3 \mathrm{vbf}$ & 4iv1 \\
\hline R.m.s.d. from final AiV1 structure $\dagger(\AA)$ & 1.64 & 1.64 & 1.64 & 1.60 & 1.63 & 1.53 & 1.76 & 1.62 & 1.57 & 1.72 \\
\hline Fraction of aligned residues $\ddagger$ (\%) & 70 & 67 & 65 & 64 & 69 & 69 & 79 & 66 & 65 & 79 \\
\hline Sequence identity to AiV1 (\%) & 16 & 16 & 15 & 16 & 19 & 19 & 20 & 15 & 17 & 18 \\
\hline
\end{tabular}

$\dagger$ R.m.s. deviations of superimposed $\mathrm{C}^{\alpha}$ atoms of the respective structures from the final AiV1 model. $\$$ Percentage of available amino-acid residues used for the calculations.

icosahedral symmetry with the cubic 23 symmetry resulted in the crystallographic asymmetric unit containing five icosahedral asymmetric units (1/12 of a virus particle).

Based on the orientation of the icosahedral particle and the crystal-packing considerations, the virus particle had to be positioned with its centre at the origin of the unit cell. Because of the body centring, there is another virus particle in the centre of the unit cell (Supplementary Fig. S2b).

3.4. Detwinning of the measured intensities based on the molecular-replacement model and NCS averaging

Detwinning of the diffraction data collected from crystals affected by perfect hemihedral twinning $(\alpha=0.5)$ cannot be based on comparisons of the observed reflection intensities (see equations 3 and 4; Yeates \& Fam, 1999; Yeates, 1997). However, a simulated twinned data set can be calculated if a model of the structure is available by summing the intensities of the reflections derived from the model with the intensities of the reflections related by the twinning operator. The ratio between the two twin-related intensities can be used to detwin the corresponding measured intensities. Thus, a known structure can supply the information necessary to detwin data affected by perfect hemihedral twinning. The detwinning procedure was performed using the following steps.

In a preparation step, $\mathrm{PDB}$ models of ten picornaviruses (PDB entries 1bev, 1cov, 1ev1, 1hxs, 1tmf, 2mev, 2wff, 2x5i, $3 v b f$ and 4iv1) were positioned in the unit cell according to the molecular-replacement solution described above (Muckelbauer et al., 1995; Smyth et al., 1995; Filman et al., 1998; Miller et al., 2001; Luo et al., 1992; Krishnaswamy \& Rossmann, 1990; Tuthill et al., 2009; Plevka et al., 2010; Wang et al., 2012; Porta et al., 2013). CNS was used to calculate the phases and structurefactor amplitudes based on the models and the five NCS operators defining the relative positions of the icosahedral asymmetric units in the crystallographic asymmetric unit (Brunger, 2007). The best molecular-replacement solution was obtained using the 1 cov structure (Table 4). The resulting CNS reflection file was converted to MTZ format using F2MTZ from CCP4 (Winn et al., 2011). This procedure provided the initial model-derived structure-factor amplitudes and phases. 
The following procedure was then used iteratively.

(i) The twin symmetry $(k, h,-l)$ was used to generate a version of the calculated structure-factor amplitudes rotated $180^{\circ}$ around the [110] axis, corresponding to the second twin domain, using REINDEX from $C C P 4$. The reflections were sorted according to the $C C P 4 h, k, l$ convention using $C A D$ (Winn et al., 2011).

(ii) The calculated structure-factor amplitudes of both of the twin domains were squared to obtain estimates of the reflection intensities. A twinning 'portion' was calculated for each reflection based on (5).

$$
\mathrm{Tw}_{h k l}^{\text {portion }}=I_{h k l} /\left(I_{h k l}+I_{k h-l}\right)
$$

Please note that each reflection had a different twinning portion. This is in contrast to the twinning fraction $\alpha$, discussed above, that characterizes the ratio of the twin domains, which is the same for all reflections from a particular data set affected by hemihedral twinning. The detwinned intensity of each reflection was calculated by multiplying the observed intensity value by the corresponding twinning portion (6). The detwinning was performed using SFTOOLS (Winn et al., 2011).

$$
I_{h k l}^{\text {detwinned }}=\left(\mathrm{Tw}_{h k l}^{\text {portion }}\right)\left(I_{h k l}^{\text {observed }}\right) .
$$

(iii) The detwinned intensities were converted to structurefactor amplitudes using TRUNCATE (Winn et al., 2011).

(iv) The phases and the structure-factor amplitudes calculated from the model were combined with the detwinned structure-factor amplitudes using SFTOOLS. The structurefactor amplitudes calculated from the model were scaled to the detwinned structure-factor amplitudes using RSTATS (Winn et al., 2011). RSTATS also produced a scaling $R$ factor and correlation coefficient comparing the scaled $\left|F_{\text {calc }}\right|$ and $\left|F_{\text {obs }}^{\text {detwinned }}\right|$, which enabled the agreement between the observed and the model-derived data to be monitored.

(v) An electron-density map $\left(2\left|F_{\text {obs }}^{\text {detwinned }}\right|-\left|F_{\text {calc }}\right|\right), \varphi_{\text {calc }}$ was calculated using FFT (Winn et al., 2011).

(vi) The electron-density map was averaged according to the fivefold NCS using $A V E$ from the Uppsala Software Factory package (Kleywegt \& Read, 1997). Two masks were used sequentially for the electron-density averaging. An initial mask was calculated based on the structure of Bovine enterovirus (BEV; PDB entry 1bev) by including all voxels within $5 \AA$ of any atom of the model using MAMA (Kleywegt \& Jones, 1999; Smyth et al., 1995). After ten cycles, the mask derived from the BEV model was replaced with a correlation map-based mask (see below for a description of the preparation of the correlation map-based mask).

(vii) Improved structure-factor amplitudes and phases were calculated from the averaged map using SFALL (Winn et al., 2011).

(viii) The procedure was cyclically repeated 30 times from step (i).
3.5. Model bias introduced by the detwinning procedure and its mitigation by NCS map averaging

An electron-density map calculated using the detwinned structure-factor amplitudes $\left(2\left|F_{\text {obs }}^{\text {detwinned }}\right|-\left|F_{\text {calc }}\right|\right)$ and phases $\varphi_{\text {calc }}$ is affected by more extensive model bias than is present in the map calculated from data that are not twinned $\left(2\left|F_{\text {obs }}\right|-\right.$ $\left.\left|F_{\text {calc }}\right|\right), \varphi_{\text {calc }}$. The additional model bias is owing to the application of the twinning portions that are derived from the molecular-replacement model (equations 5 and 6). The differences between the molecular-replacement model and the actual crystallized structure result in errors in the twinning portions that subsequently introduce errors into the detwinned amplitudes $\left|F_{\text {obs }}^{\text {detwinned }}\right|$. Thus, the detwinning procedure limits the amount of information in the $\left(2\left|F_{\text {obs }}^{\text {detwinned }}\right|-\left|F_{\text {calc }}\right|\right), \varphi_{\text {calc }}$ map calculation that is provided by the $\left|F_{\text {obs }}\right|$ values. In addition, as in the standard molecularreplacement map calculation, the differences between the model and the crystallized structure result in errors in phases $\left(\varphi_{\text {calc }}\right)$ that introduce model bias. However, the NCS averaging effectively increases the observed data redundancy, since volumes of the NCS-related molecules are forced to have the same electron-density distributions. Differences between the NCS-related positions owing to errors in the twinning portions and phases are removed by the NCS averaging. The resulting averaged electron-density map can be used to calculate improved phases and twinning portions that better resemble the crystallized structure. The averaging procedure combined with detwinning resulted in an improvement in the $R$-factor value on comparing $\left|F_{\text {calc }}\right|$ with $\left|F_{\text {obs }}^{\text {detwinned }}\right|$ (Fig. $2 a$ ).

\subsection{Optimization of the NCS averaging parameters}

The NCS rotation-translation operators and the shape of the averaging mask have to be accurately determined for effective use of NCS averaging to improve the twinning portions and phases. For the AiV1 crystals, the fivefold NCS operators were determined by aligning the icosahedral symmetry with the 23 cubic symmetry of the crystal (Figs. $1 a$, $1 b$ and $1 d$ and Supplementary Fig. S $2 b)$. However, the initial averaging mask derived by including all voxels within $5 \AA$ of any atom of the BEV model could have had an incorrect shape because of the differences in the capsids of BEV and AiV1. Therefore, a correlation map-based mask (Vellieux et al., 1995) was prepared using the following steps. A correlation map was calculated with a voxel size of $4.5 \AA$. Each voxel of the correlation map corresponded to 265 voxels of the AiV1 electron-density map (voxel size $0.7 \AA$ ). Each voxel in the correlation map was assigned a value of the correlation coefficient calculated by comparing the corresponding 265 electron-density map values of voxels in the five NCS-related volumes. The correlation map was calculated using COMA (Kleywegt \& Jones, 1999). A cutoff value of 0.65 was used for including the voxels from the correlation map into the correlation map-based mask. The use of the correlation mapbased mask resulted in a decreased $R$ factor comparing $\left|F_{\text {calc }}\right|$ and $\left|F_{\text {obs }}^{\text {detwinned }}\right|$ relative to when the BEV-derived mask was used (Fig. 2a). Ex post, we could also show that the use of the 
correlation map-based mask resulted in decreased phase differences from the phases derived from the final AiV1 model

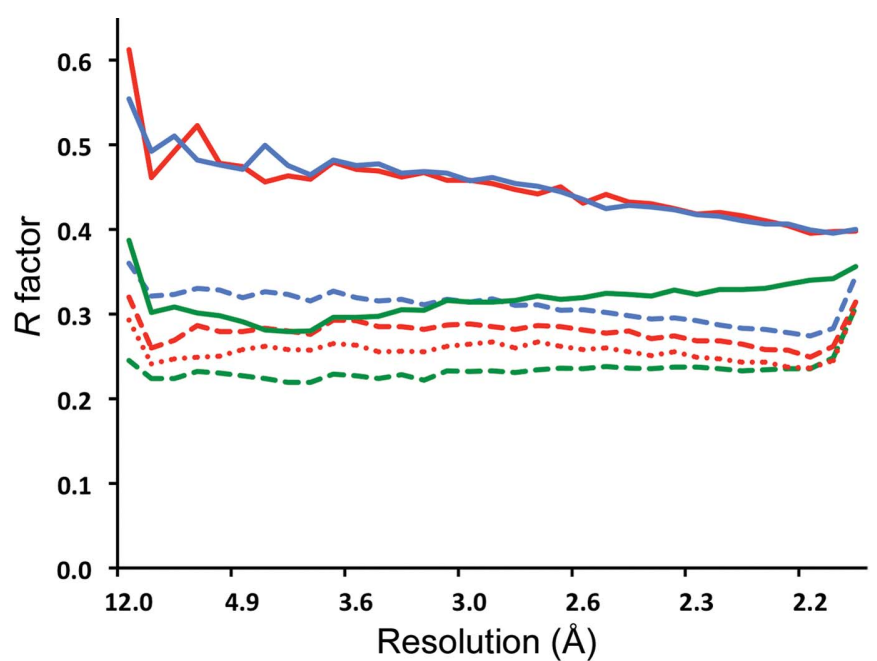

(a)

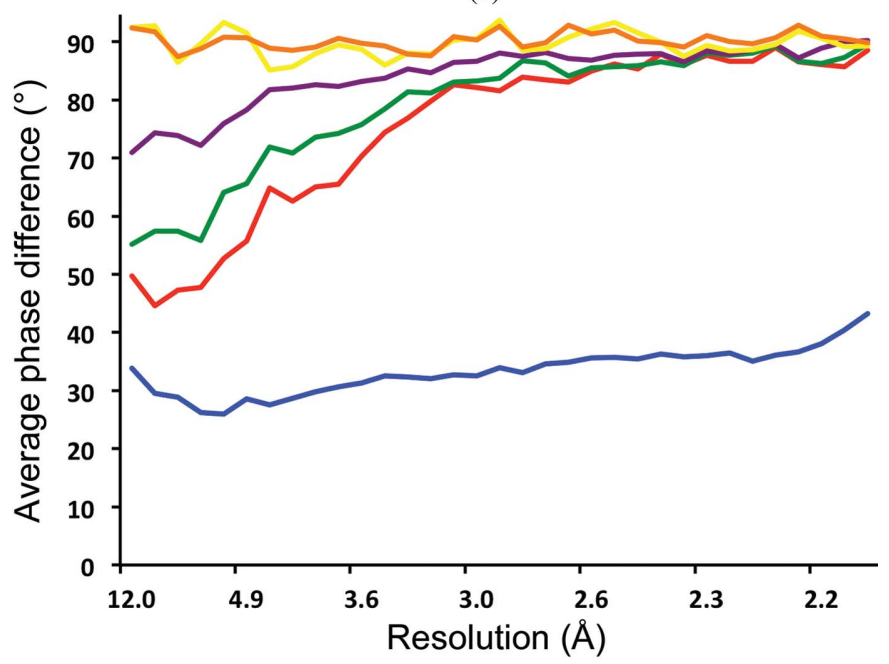

(b)

Figure 2

(a) Crystallographic $R$ factors comparing $\left|F_{\text {obs }}^{\text {detwinned }}\right|$ and $\left|F_{\text {calc }}\right|$ as a function of resolution at different stages of $\mathrm{AiV} 1$ structure determination. The $R$ factor comparing $\left|F_{\text {obs }}^{\text {detwinned }}\right|$ and $\left|F_{\text {calc }}\right|$ calculated from the BEV model converted to polyalanine is shown as a continuous red line, the $R$ factor after refinement by ten cycles of NCS averaging using the BEVderived mask is shown as a dashed red line and the $R$ factor after 30 cycles of NCS averaging using the correlation map-based mask is shown as a dotted red line. The $R$ factor comparing $\left|F_{\text {obs }}^{\text {detwinned }}\right|$ and $\left|F_{\text {calc }}\right|$ calculated from the final AiV1 model is shown as a continuous green line and the $R$ factor after ten cycles of NCS averaging as a dashed green line. The $R$ factor comparing $\left|F_{\text {obs }}^{\text {detwinned }}\right|$ and $\left|F_{\text {calc }}\right|$ calculated from the $\varphi \mathrm{Cb} 5$ structure is shown as a continuous blue line and the $R$ factor after ten cycles of NCS averaging is shown as a dashed blue line. (b) Phase-difference plots comparing phases at various stages of structure determination with phases derived from the final AiV1 structure and refined by 30 cycles of NCS averaging. Phase differences were calculated in narrow resolution bins and plotted against resolution. The average phase difference of phases of the BEV model are shown as a violet line, of the BEV model refined by ten cycles of the NCS averaging using the BEV-derived averaging mask as a green line, of the BEV model refined by 30 cycles of the NCS averaging using the correlation-map based mask as a red line, of the $\varphi \mathrm{Cb} 5$ model as an orange line, of the $\varphi \mathrm{Cb} 5$ model refined by ten cycles of the NCS averaging as a yellow line and of the final AiV1 structure as a blue line. (See text for further details.)
(Fig. 2b). This indicated that the correlation map could be used to improve the shape of the mask used for NCS averaging, even for crystals affected by perfect hemihedral twinning.

\subsection{Quality of electron-density maps}

The interpretability of an electron-density map calculated from the detwinned structure-factor amplitudes depends on the similarity of the phasing/detwinning model to the crystallized structure. Several maps with a varying utility for model building were obtained in the course of the AiV1 structure determination. The maps were closely inspected in terms of the presence of features corresponding to the AiV1 structure that were different from the molecular-replacement model. The quality of the phases used in map calculations was checked ex post by calculating phase-difference plots comparing the phases used to calculate maps at different stages of structure determination with phases from the final AiV1 model refined by ten cycles of NCS averaging (Fig. 2b). The initial $\left(2\left|F_{\text {obs }}^{\text {detwinned }}\right|-\left|F_{\text {calc }}\right|\right), \varphi_{\text {calc }}$ map was calculated based on the phases and the twinning portions derived from the BEV model converted to a polyalanine chain. The map was strongly affected by model bias and did not show any features other than those of the BEV structure (Figs. $2 b$ and $3 a$ ). The phases and the twinning portions were refined by ten cycles of NCS averaging (Figs. $2 b$ and $3 b$ ). The resulting map was used to determine the correlation map-based mask. To utilize the availability of the numerous picornavirus models determined to atomic resolutions, a map was calculated by combining ten picornavirus models in order to calculate the initial phases and the twinning portions. Subsequently, the phases and the twinning portions were refined by 30 cycles of NCS averaging using the correlation map-based mask (Figs. $2 b, 3 c$ and $2 d$ ). The resulting map was of sufficient quality to enable an initial manual model build. During the model building the electron-density maps were frequently recalculated with the initial phases derived from the latest model followed by 30 cycles of NCS averaging (Figs. $2 b$ and $3 e$ ). The maps calculated using the phases and the twinning portions refined by NCS averaging exhibited clearer features than the map calculated with the phases and the twinning portions derived from the final AiV1 model (Figs. $2 b$ and $3 f$ ).

\subsection{Convergence radius of the detwinning procedure}

To test the limits of the convergence radius of the detwinning approach towards the correct phase solution, a molecularreplacement model with a different structure from that of the picornavirus capsid (bacteriophage $\varphi \mathrm{Cb} 5$; PDB entry 2w4y; Plevka et al., 2009) was tested. A map calculated with the phases and the twinning portions derived from the $\varphi \mathrm{Cb} 5 \mathrm{PDB}$ model was affected by strong model bias and exhibited features of the $\varphi \mathrm{Cb} 5$ structure (Figs. $2 b$ and $3 g$ ). After 30 cycles of NCS averaging, the electron-density map did not resemble $\varphi \mathrm{Cb} 5$; however, the map was uninterpretable (Figs. $2 b$ and $3 h$ ). This indicated that the combination of molecular-replacement model-based detwinning with NCS 
averaging is a relatively safe approach for the removal of model bias because the calculation either converged to the correct structure (if the initial phasing model was sufficiently similar to the crystallized structure) or produced an uninterpretable map (if the initial model was too different from the crystallized structure). The $R$ factors comparing $\left|F_{\mathrm{obs}}^{\text {detwinned }}\right|$ with $\left|F_{\text {calc }}\right|$ were similar for the $\varphi \mathrm{Cb} 5$ and BEV structures (Fig. 2a). However, the NCS averaging produced a lower $R$ factor in the phasing attempt initiated with the BEV model (Fig. 2a). The quality of the phases obtained from the BEV and $\varphi \mathrm{Cb} 5$ models and the subsequent NCS averaging refinements was evaluated ex post by comparing the phases

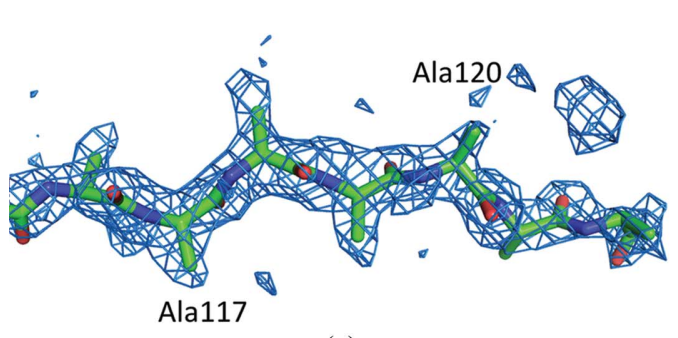

(a)

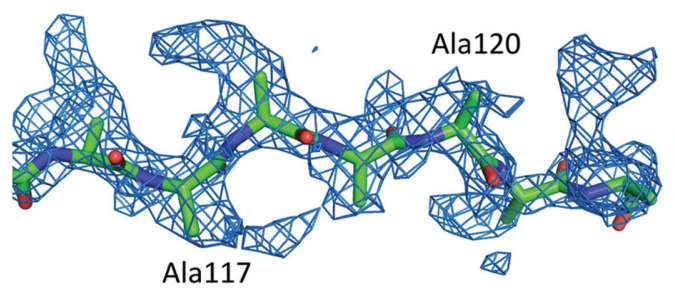

(c)

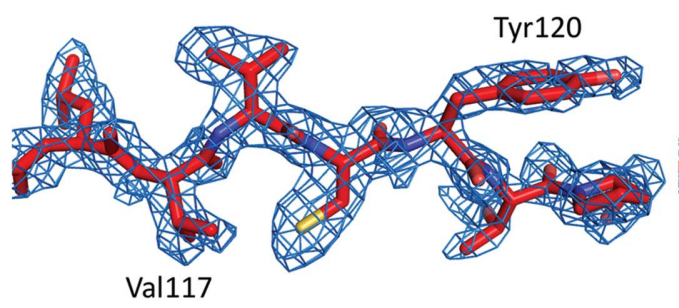

(e)

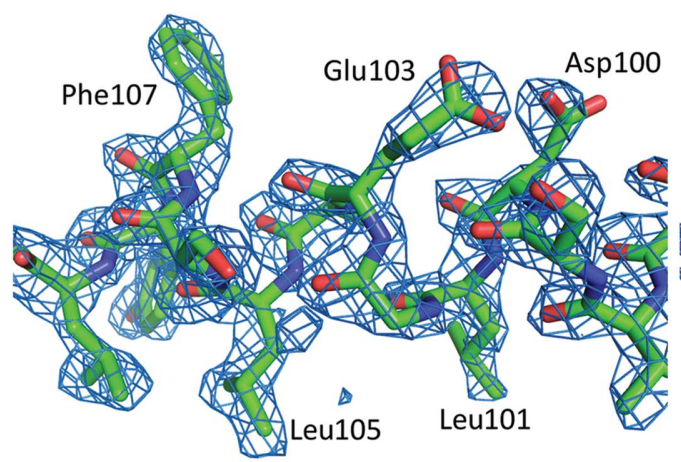

$(g)$

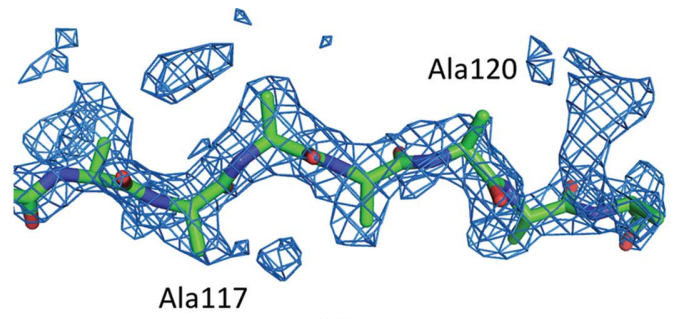

(b)

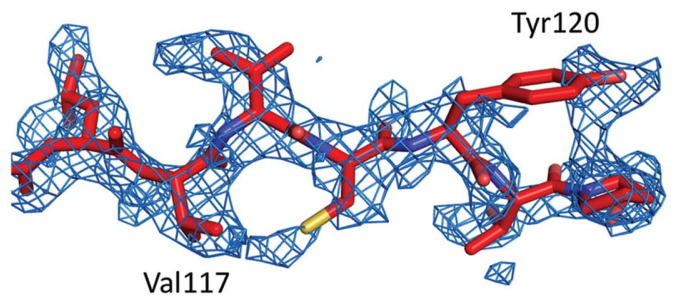

(d)

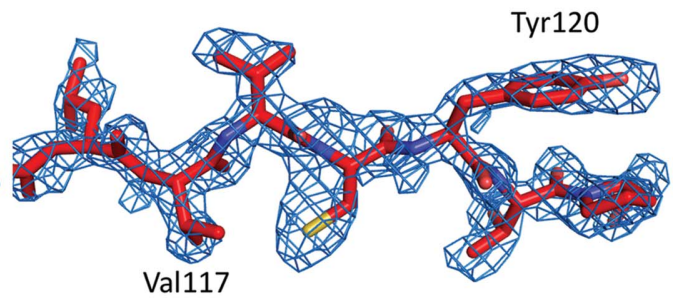

$(f)$

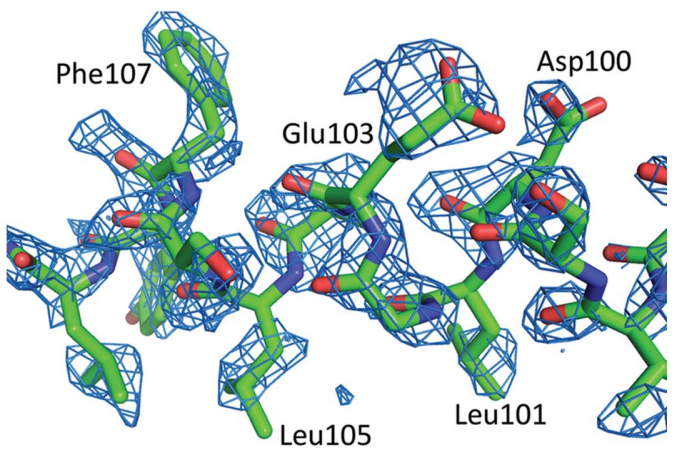

(h)

Figure 3

Comparison of $\left(2\left|F_{\text {obs }}^{\text {detwinned }}\right|-\left|F_{\text {calc }}\right|\right), \varphi_{\text {calc }}$ electron-density maps at various stages of AiV1 structure determination. (a) Electron-density map calculated using the phases and twinning portions derived from the BEV model converted to a polyalanine chain. The BEV model converted to polyalanine is shown in stick representation with the $\mathrm{C}$ atoms coloured green. $(b)$ Electron-density map calculated using the phases and twinning portions from the BEV model converted to polyalanine and refined by ten cycles of NCS averaging using the BEV-derived mask. The BEV model converted to polyalanine is shown in stick representation with the $\mathrm{C}$ atoms coloured green. (c) Electron-density map calculated using the phases and twinning portions from the BEV model converted to polyalanine and refined by 30 cycles of NCS averaging using the correlation map-derived mask. See $\$ 3.6$ for details of the mask preparation. The BEV model converted to polyalanine is shown in stick representation with the $\mathrm{C}$ atoms coloured green. $(d)$ The same electron-density map as in $(c)$ with the final AiV1 model shown in stick representation with the $\mathrm{C}$ atoms coloured red. (e) An electron-density map calculated using the phases and the twinning portions derived from the final AiV1 model. The final AiV1 model is shown in stick representation with the C atoms coloured red. $(f)$ Electron-density map calculated using the phases and twinning portions derived from the final AiV1 structure and refined by ten cycles of NCS averaging. The final AiV1 model is shown in stick representation with the $\mathrm{C}$ atoms coloured red. $(g)$ Electron-density map calculated using the phases and twinning portions derived from the $\varphi \mathrm{Cb} 5$ structure. The model of $\varphi \mathrm{Cb} 5$ is shown in stick representation with the $\mathrm{C}$ atoms coloured green. ( $h$ ) Electron-density map calculated using the phases and twinning portions from the $\varphi \mathrm{Cb} 5$ structure and refined by ten cycles of NCS averaging. The model of $\varphi \mathrm{Cb} 5$ is shown in stick representation with the $\mathrm{C}$ atoms coloured green. 
calculated from the final AiV1 model and refined by 30 cycles of NCS averaging (Fig. $2 b$ ).

The effectiveness of NCS averaging in obtaining the correct phases and the twinning portions was tested by calculating an OMIT map with the phases and the twinning portions derived from the final AiV1 model with residues $117-120$ of VP2 deleted. The resulting OMIT map lacked the electron density corresponding to the deleted residues (Fig. 4a). However, the electron density of the deleted residues could be recovered by ten cycles of NCS averaging (Fig. $4 b$ ).

3.9. Size of the twin domains in comparison to the coherence length of the X-ray beam

The crystallization conditions from which the twinned $I 23$ crystal was obtained also produced crystals with apparent space group $\mathrm{P4}_{2} 32$. The unit-cell size of the $P 4_{2} 32$ crystal ( $a=351.1 \AA$ ) was nearly identical to the unit cell of the $I 23$ crystal $(a=350.8 \AA)$. A statistical analysis of the reflection intensities from the $\mathrm{P}_{2} 32$ data set produced values that were even lower than the values expected for perfectly hemihedrally twinned data (Table 2). The native Patterson function calculated from the $\mathrm{P}_{2} 32$ data did not contain any large offorigin peaks. We interpret the statistics by proposing that the $P 4_{2} 32$ crystal was built from the same domains as the $I 23$ twinned crystals; however, in the $P_{2} 32$ crystal the domains were smaller than the coherent length of the X-ray beam. Thus, the $\mathrm{X}$-rays diffracted from the individual domains in the different orientations interacted as waves. The complex interaction of the diffracted X-rays might have resulted in the observed low twinning statistics (Table 2). This is in contrast to twinning, where the crystal domains are large relative to the coherence length and the diffracted beams sum their intensities. The I23 AiV1 unit cell contains one particle in the

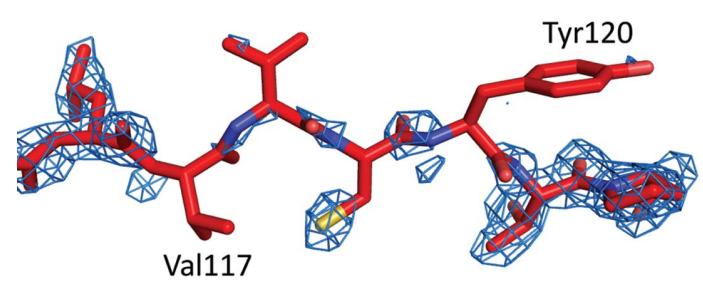

(a)

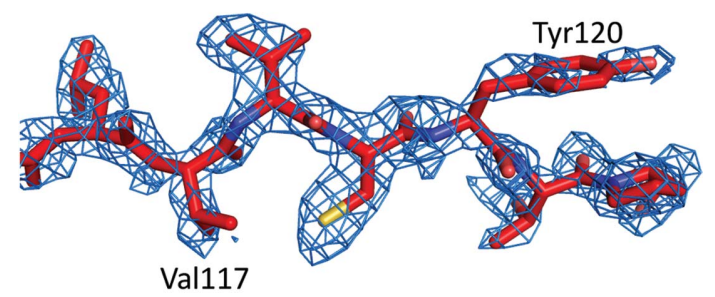

(b)

Figure 4

Electron density of a missing part of the structure can be recovered by a combination of the detwinning procedure and NCS averaging. (a) An OMIT $\left(2\left|F_{\text {obs }}^{\text {detwinned }}\right|-\left|F_{\text {calc }}\right|\right), \varphi_{\text {calc }}$ map calculated using the phases and the twinning portions derived from the final AiV1 model with deleted residues 117-120 of VP2. (b) An electron-density map for the missing part was recovered by ten cycles of NCS averaging. corner (fractional coordinates $0,0,0)$ and another particle with an identical orientation in the centre $(0.5,0.5,0.5)$. The $P 4_{2} 32$ unit cell might also contain two virus particles located at $(0,0,0)$ and $(0.5,0.5,0.5)$; however, the particle in the centre is rotated $90^{\circ}$ relative to the particle in the corner (Supplementary Fig. S2c). The possibility of accommodating both of the $90^{\circ}$ rotation-related particle orientations in the AiV1 crystal is consistent with the hemihedral twinning observed in the $I 23$ crystal. The $P 4_{2} 32$ data set did not produce an interpretable electron-density map even when it was phased using the final AiV1 model.

\subsection{Model quality}

The electron-density map obtained after 30 cycles of realspace NCS averaging combined with detwinning was interpretable for most of the AiV1 structure. However, some regions, including the surface loops of VP1 located close to the icosahedral fivefold axes and the N-terminal arms of the capsid proteins located on the inside of the capsid, were difficult to interpret. The electron-density map of these parts became clearer when intermediate AiV1 models were used to calculate the initial phases in the detwinning procedure. For model construction, manual model building using $O$ and $C o o t$ (Jones et al., 1990; Emsley et al., 2010) was alternated with refinement using $C N S$ with the input files minimize_twin.inp and bindividual_twin.inp (Brunger, 2007).

The final AiV1 model includes residues 1-83 and 88-233 of VP1, residues 13-55, 64-75 and 112-370 of VP2 and residues 1-220 of VP3 together with 173 water molecules within one icosahedral asymmetric unit of the virion. The final crystallographic $R$ factor (0.33) is high relative to the $R_{\text {merge }}(0.166)$ of the $2.1 \AA$ resolution data set (Table 3 ). The high $R$ value might be owing to the complicated refinement using data affected by perfect hemihedral twinning.

The $R_{\text {free }}$ factor (Brünger, 1992) was not calculated because it was not possible to select a set of reflections that would be independent of the reflections in the part of the data set used for the refinement (Fabiola et al., 2006; Kleywegt \& Brünger, 1996). To avoid correlations between working and free sets, the free-set reflections would need to be selected within fivefold NCS-related groups. In addition, both of the twin operator-related reflections would have to be included in the test set. However, it has been shown previously that it is not sufficient to select the $R_{\text {free }}$ set in thin resolution shells because the reflections are correlated not only within the resolution shell but also with the neighbouring reflections of higher and lower resolution (Chen et al., 1999). Thus, if calculated, $R_{\text {free }}$ would be very similar to the $R$ value owing to the fivefold NCS and the twin operator present in the diffraction data (Kleywegt \& Brünger, 1996). Instead of using $R_{\text {free, }}$ the optimal weight of the X-ray refinement function relative to the energy minimization of the model was determined by checking the geometry of the model based on the r.m.s.d. of bond angles and lengths (Kleywegt, 2000). 
3.11. Utility of the detwinning procedure for other perfectly hemihedrally twinned data sets

The determination of a macromolecular structure from diffraction data affected by perfect hemihedral twinning is challenging because (i) the data cannot be detwinned unless a sufficiently similar model is available and (ii) even if a suitable model is available, the calculated electron-density map is affected by more extensive model bias than with untwinned data. However, here we show that it is possible to detwin perfectly hemihedrally twinned data and solve the structure in the presence of fivefold NCS. The best available molecularreplacement model (PDB entry 1cov) had 16\% sequence identity and a $1.6 \AA$ r.m.s.d. of $\mathrm{C}^{\alpha}$ atoms for the $67 \%$ of the AiV1 residues that could be aligned (Table 4). The NCS averaging procedure reduced the model bias introduced by the differences between the molecular-replacement model and the crystallized structure. In the test case of bacteriophage $\varphi \mathrm{Cb} 5$, the procedure failed and produced an uninterpretable electron-density map. This functions as a safety check preventing the construction of structures biased towards the molecular-replacement model. The approach presented here could be used for other crystals affected by perfect hemihedral twinning that contain at least fivefold NCS.

\section{Acknowledgements}

We wish to thank the scientists at beamline I03 of Diamond Light Source and at the PROXIMA1 and PROXIMA2A beamlines at Synchrotron SOLEIL for their help with crystal screening and data collection. We wish to thank Dr Jana Moravcová for her help with the preparation of the manuscript for submission. We wish to thank Dr Lenka Pálková for help with AiV1 growth and purification. We wish to thank Dr A. Michael Lindberg for providing the AiV1 for the initial infection. The research leading to these results has received funding from the European Research Council under the European Union's Seventh Framework Programme (FP/20072013)/ERC Grant Agreement No. 355855 and from EMBO installation grant \#3041 to PP. This research was carried out under the project CEITEC 2020 (LQ1601) with financial support from the Ministry of Education, Youth and Sports of the Czech Republic under the National Sustainability Program II.

\section{References}

Brünger, A. T. (1992). Nature (London), 355, 472-475.

Brunger, A. T. (2007). Nature Protoc. 2, 2728-2733.

Chandra, N., Acharya, K. R. \& Moody, P. C. E. (1999). Acta Cryst. D55, 1750-1758.
Chen, V. B., Arendall, W. B., Headd, J. J., Keedy, D. A., Immormino, R. M., Kapral, G. J., Murray, L. W., Richardson, J. S. \& Richardson, D. C. (2010). Acta Cryst. D66, 12-21.

Chen, Z., Blanc, E. \& Chapman, M. S. (1999). Acta Cryst. D55, 219-224.

Emsley, P., Lohkamp, B., Scott, W. G. \& Cowtan, K. (2010). Acta Cryst. D66, 486-501.

Evans, P. (2006). Acta Cryst. D62, 72-82.

Fabiola, F., Korostelev, A. \& Chapman, M. S. (2006). Acta Cryst. D62, 227-238.

Filman, D. J., Wien, M. W., Cunningham, J. A., Bergelson, J. M. \& Hogle, J. M. (1998). Acta Cryst. D54, 1261-1272.

Grainger, C. T. (1969). Acta Cryst. A25, 427-434.

Helliwell, J. R. (2008). Crystallogr. Rev. 14, 189-250.

Jones, T. A., Bergdoll, M. \& Kjeldgaard, M. (1990). Crystallographic and Modeling Methods in Molecular Design, edited by C. E. Bugg \& S. E. Ealick, pp. 189-199. New York: Springer-Verlag.

Kabsch, W. (2010). Acta Cryst. D66, 125-132.

Kleywegt, G. J. (2000). Acta Cryst. D56, 249-265.

Kleywegt, G. J. \& Brünger, A. T. (1996). Structure, 4, 897-904.

Kleywegt, G. J. \& Jones, T. A. (1999). Acta Cryst. D55, 941-944.

Kleywegt, G. J. \& Read, R. J. (1997). Structure, 5, 1557-1569.

Krishnaswamy, S. \& Rossmann, M. G. (1990). J. Mol. Biol. 211 , 803-844.

Luo, M., He, C., Toth, K. S., Zhang, C. X. \& Lipton, H. L. (1992). Proc. Natl Acad. Sci. USA, 89, 2409-2413.

Miller, S. T., Hogle, J. M. \& Filman, D. J. (2001). J. Mol. Biol. 307, 499-512.

Muckelbauer, J. K., Kremer, M., Minor, I., Tong, L., Zlotnick, A., Johnson, J. E. \& Rossmann, M. G. (1995). Acta Cryst. D51, 871887.

Padilla, J. E. \& Yeates, T. O. (2003). Acta Cryst. D59, 1124-1130.

Parsons, S. (2003). Acta Cryst. D59, 1995-2003.

Plevka, P., Hafenstein, S., Harris, K. G., Cifuente, J. O., Zhang, Y., Bowman, V. D., Chipman, P. R., Bator, C. M., Lin, F., Medof, M. E. \& Rossmann, M. G. (2010). J. Virol. 84, 12665-12674.

Plevka, P., Kazaks, A., Voronkova, T., Kotelovica, S., Dishlers, A., Liljas, L. \& Tars, K. (2009). J. Mol. Biol. 391, 635-647.

Porta, C., Kotecha, A., Burman, A., Jackson, T., Ren, J., Loureiro, S., Jones, I. M., Fry, E. E., Stuart, D. I. \& Charleston, B. (2013). PLoS Pathog. 9, e1003255.

Redinbo, M. R. \& Yeates, T. O. (1993). Acta Cryst. D49, 375-380.

Smyth, M., Tate, J., Hoey, E., Lyons, C., Martin, S. \& Stuart, D. (1995). Nature Struct. Biol. 2, 224-231.

Tong, L. \& Rossmann, M. G. (1990). Acta Cryst. A46, 783-792.

Tuthill, T. J., Harlos, K., Walter, T. S., Knowles, N. J., Groppelli, E., Rowlands, D. J., Stuart, D. I. \& Fry, E. E. (2009). PLoS Pathog. 5, e1000620.

Vellieux, F. M. D. A. P., Hunt, J. F., Roy, S. \& Read, R. J. (1995). J. Appl. Cryst. 28, 347-351.

Wang, X. et al. (2012). Nature Struct. Mol. Biol. 19, 424-429.

Winn, M. D. et al. (2011). Acta Cryst. D67, 235-242.

Yamashita, T., Kobayashi, S., Sakae, K., Nakata, S., Chiba, S., Ishihara, Y. \& Isomura, S. (1991). J. Infect. Dis. 164, 954-957.

Yamashita, T., Sakae, K., Tsuzuki, H., Suzuki, Y., Ishikawa, N., Takeda, N., Miyamura, T. \& Yamazaki, S. (1998). J. Virol. 72, 84088412.

Yeates, T. O. (1997). Methods Enzymol. 276, 344-358.

Yeates, T. O. \& Fam, B. C. (1999). Structure, 7, R25-R29. 